(205)

\title{
A Study of the Relationship between Ecological Network Patch metrics and Landscape connectivity; with reference to case of Colombo Wetlands
}

\author{
Ekanayake H.E.M.W.G.M.K. ${ }^{1 *}$,Dharmasena J. ${ }^{2}$ \\ ${ }^{1}$ Landscape degree Programme, University of Moratuwa, Sri Lanka \\ ${ }^{2}$ Department of Architecture, University of Moratuwa, Sri Lanka \\ *maheshi92ke@gmail.com
}

\begin{abstract}
Landscape fragmentation and habitat loss are emergent issues in Sri Lanka, which is a result of rapid urban development and inadequate concern of managing Landscape connectivity. Inland Wetlands and forests are the most valuable ecosystems effected from the fragmentation. Considering those habitats, Wetlands are among the most productive, active, diverse, and beneficial ecosystems in nature threatened by the urbanization. Therefore, this study aimed to introduce spatial strategies to locate landscape developments to restore landscape connectivity; referring to Colombo wetlands. Though the fragmentation causes alteration of wetland structure, fragments does serves to landscape connectivity functioning as an ecological network. Therefore the study aims to understand how the habitat/ ecological network patch metrics contribute to landscape connectivity. Two main methods used; to calculate the patch metrics; Patch area, Total edge, Perimeter-area ratio, Core area index and Inter-patch distances; GIS data have been used. Further, GIS enables least-cost path tool to be used to measure the Landscape connectivity and calculate number of species flow paths per wetland patch. With the data analysis, the study investigated the relationship between the patch metrics and the landscape connectivity. Referring to the findings, the study discussed; increasing the patch area, maintaining a mean perimeter-area ratio or core area index, reducing the inter patch distances could enhance landscape connectivity within in the urban wetland habitat patches. At the end, the study introduces strategies for Landscape architects to select most suitable locations to implement ecological based landscape developments adjacent to the existing urban habitat in order to enhance patch metrics and to restore the landscape connectivity
\end{abstract}

Keywords: Landscape fragmentation, Ecological networks, Patch metrics, Landscape connectivity 